\title{
Periodic Orbits, Spectral Oscillations, Scaling, and Vacuum Energy: Beyond HaMiDeW
}

\author{
S. A. Fulling a
}

${ }^{a}$ Mathematics Department, Texas A\&M University, College Station, TX 77843-3368, U.S.A.

Oscillations in eigenvalue density are associated with closed orbits of the corresponding classical (or geometrical optics) system. Although invisible in the heat-kernel expansion, these features determine the nonlocal parts of propagators, including the Casimir energy. I review some classic work, discuss the connection with vacuum energy, and show that when the coupling constant is varied with the energy, the periodic-orbit theory for generic quantum systems regains the clarity and simplicity that it always had for the wave equation in a cavity.

While quantum gravity people were learning about the heat kernel, atomic physicists were progressing beyond it.

The key property of the heat kernel expansion is its locality: the coefficients $a_{n}(x)$ are completely determined by the metric and potentials in a small neighborhood of $x$. This makes it easy to compute and universal in its applications to renormalization of ultraviolet divergences. Its formal inverse Laplace transform is an "averaged" spectral density [1-3] that is equally local and universal, insensitive to the detailed spacings of the eigenvalues (if the spectrum is discrete at all).

The interesting part of renormalization is what is left behind when the counterterms are subtracted. For example, vacuum (Casimir) energy can be calculated from a two-point function (a kernel for the wave equation) by subtracting universal singular terms. The wave kernel and the vacuum energy are nonlocal; they reflect boundary conditions, global topology, presence of periodic or closed classical orbits, and whether the dynamics is chaotic or integrable. This global geometrical information is encoded in the fine structure of the spectrum.

More precisely, let $T(t, x, y)$ be the integral kernel of $e^{-t \sqrt{H}}$, where $H$ is some positive selfadjoint second-order differential operator. (This "cylinder kernel" is analytically more tractable than kernels of wave (e.g., $e^{-i t \sqrt{H}}$ ) or Schrödinger $\left(e^{-i t H}\right)$ operators, but retains at least some of their nonlocal information [4].) Assume for now a discrete spectrum, with $H \phi_{j}=\omega_{j}^{2} \phi_{j},\left\|\phi_{j}\right\|=1$. Then

$$
\begin{gathered}
T(t, x, y)=\sum_{j=1}^{\infty} e^{-t \omega_{j}} \phi_{j}(x) \phi_{j}(y)^{*}, \\
\int T(t, x, x) d x=\sum_{j=1}^{\infty} e^{-t \omega_{j}}
\end{gathered}
$$

On the other hand, for a scalar field theory with field equation $\frac{\partial^{2} \phi}{\partial t^{2}}=-H \phi$ the vacuum energy density is formally

$T_{00}=\left\langle\frac{1}{2}\left[\left(\frac{\partial \phi}{\partial t}\right)^{2}+\cdots\right]\right\rangle$,

where the omitted terms involve spatial derivatives and are equivalent modulo integration by parts to $\phi H \phi$. It follows that

$$
\begin{gathered}
T_{00}(x)=\frac{1}{4} \sum_{j=1}^{\infty}\left[\omega_{j}\left|\phi_{j}(x)\right|^{2}+\omega_{j}^{-1} \cdots\right], \\
\int T_{00} d x=\frac{1}{2} \sum_{j=1}^{\infty} \omega_{j} .
\end{gathered}
$$

Comparing (1) and (2), we see that

$-\frac{1}{2} \frac{\partial}{\partial t} T(t, x, x)$

is a regularization of the time-derivative terms of $2 T_{00}(x)$ by an exponential cutoff, and its integral performs the same service for the total vacuum energy. (The space-derivative terms of $T_{00}$ are related to the kernel of $H^{-1 / 2} e^{-t \sqrt{H}}$. These two 
cylinder kernels can be regarded as Green functions for elliptic boundary value problems with Dirichlet and Neumann data, respectively, on the surface $t=0$.)

Since $T$ is not local, it cannot be computed (even expanded at small $t$ ) by local methods. Occasionally physicists have adapted the construction of the heat kernel (for $e^{-t H}$ ) by the pseudodifferential symbolic calculus to $e^{-t \sqrt{H}}$. This yields a correct parametrix, but generally the smooth remainder in $T$ is not transcendentally small. In particular, such a calculation falsely predicts that there is no Casimir effect.

The remainder of this article summarizes [5].

We all know that in the simplest (Fourier series) models three things are intimately related: the Casimir energy, the finite spacing of the eigenvalues, and the solution by the method of images (alias Poisson summation formula) - which can be paraphrased by the observation that $\mathbf{x}$ can be connected to $\mathbf{y}$ not only directly, but also by paths that wind around the universe (or bounce off walls) many times. The connection between spectrum and classical paths has an extension to more general systems that is well known in atomic physics [6]: "The average density of states as a function of energy [is] equal to a smooth monotonic function, related to the volume occupied by the energy-shell in phase-space, plus a sum of sinusoidal oscillations. The wavelength and amplitude of each oscillation are respectively correlated with the period and the stability of a periodic orbit of the system. For given energy resolution $\Delta E$, only those periodic orbits are significant for which the period is less than $2 \pi \hbar / \Delta E . "$

The development of this subject continues actively in both mathematics and physics (e.g., $[7,8])$, but the period of greatest activity was around 1970 and can be represented by three classic papers, treating distinct problems: (1) Balian and Bloch [9], Laplacian in a bounded region; (2) Duistermaat and Guillemin [10], Laplace-Beltrami operator on a compact manifold (and generalizations); (3) Gutzwiller [11], $H=-\frac{\hbar^{2}}{2 m} \nabla^{2}+\lambda V(\mathbf{x})$ in $\mathbf{R}^{n}$. In [11] the potential $V$ is responsible for confining the particle and hence making the spectrum discrete. Thus the classical paths are solutions of the full dynamics,

$\frac{d \mathbf{x}}{d t}=\frac{\mathbf{p}}{m}, \quad \frac{d \mathbf{p}}{d t}=-\lambda \nabla V(\mathbf{x})$.

(In [10] the paths are geodesics, even when a lower-order perturbation is added to $H$.) Gutzwiller studies the Schrödinger equation while the other two deal with the wave equation, but that is unimportant: A given elliptic operator $H$ can be studied in either setting, and the two sets of orbits are in trivial one-to-one correspondence.

Any solution curve of (3) lies on an energy surface

$\frac{\mathbf{p}^{2}}{2 m}+\lambda V(\mathbf{x})=E$.

Assume that the spectrum of $H$ is discrete, at least below some threshold. The essence of the Gutzwiller trace theory is that the eigenvalue density, $\rho(E)$, contains a term

$\rho_{\gamma}(E)=a_{\gamma} \sin \left[\frac{S_{\gamma}}{\hbar}+\eta_{\gamma}\right]$

for each isolated classical periodic orbit $\gamma$ of en$\operatorname{ergy} E$, where $S_{\gamma}(E)$ is the action $\oint_{\gamma} \mathbf{p} \cdot d \mathbf{x}$. For the (possibly broken) geodesics studied in $[9,10]$, one has exactly

$S_{\gamma}(E)=2 E T_{\gamma}(E)=\sqrt{2 m E} L_{\gamma}$,

where the orbit's length $L_{\gamma}$ is independent of $E$. So, to the extent that $a_{\gamma}$ and $\eta_{\gamma}$ are constant, and with $\frac{\hbar^{2}}{2 m}=1, \rho_{\gamma}$ oscillates exactly with frequency $L_{\gamma}$ as a function of $\omega=\sqrt{E}$; also, as function of $E$ it oscillates locally with approximate frequency $T_{\gamma}$ (the classical period).

In the case (3) the situation is more subtle, because the orbits change with $E$. Of course, it is reasonable to expect that, apart from occasional bifurcations, the isolated orbits will form families indexed by $E$; for the resulting locally defined functions $\rho_{\gamma}(E)$, approximate local frequencies of oscillation can be defined. But the crisp picture associated with energy-independent geodesics is theatening to fade out. Fortunately, fixed orbits can be recovered by two strategies, or a combination of them. 
Proposition 1 (Cf. [12].) Let $\left(\mathbf{x}_{0}(t), \mathbf{p}_{0}(t)\right)$ be a solution of (3) and (4) with $\lambda=\lambda_{0}$ and $E=E_{0}$. For any $\alpha>0$ let

$\mathbf{x}(t)=\mathbf{x}_{0}(\alpha t), \quad \mathbf{p}(t)=\alpha \mathbf{p}_{0}(\alpha t)$.

Then $(\mathbf{x}(t), \mathbf{p}(t))$ satisfies (3) and (4) with

$\lambda=\alpha^{2} \lambda_{0}, \quad E=\alpha^{2} E_{0}$,

$$
T=\alpha^{-1} T_{0}, \quad S=\alpha S_{0} .
$$

Thus (5) becomes

$$
\begin{aligned}
\rho_{\gamma} & =a_{\gamma} \sin \left[\frac{S_{0}}{\hbar} \sqrt{\frac{E}{E_{0}}}+\eta_{\gamma}\right] \\
& =a_{\gamma} \sin \left(l_{\gamma} \omega+\eta_{\gamma}\right) \quad \text { in natural units, }
\end{aligned}
$$

where $l_{\gamma}=S_{0} / \sqrt{2 m\left|E_{0}\right|}=S / \sqrt{2 m|E|}$ (independent of $\alpha$ and of $E$ ) is a characteristic length of the orbit (not necessarily equal to the actual length, $\left.L_{\gamma}\right)$.

In words, the same closed orbit exists for all values of the energy, and $\rho_{\gamma}$ is a globally sinusoidal function of $\sqrt{E}$ as in the geodesic case; but this has been attained at the cost of varying the coupling constant - i.e., considering different physical systems at different energies. An alternative approach is possible when the potential has a scaling symmetry:

\section{Proposition 2 Suppose that}

$V(\beta \mathbf{x})=\beta^{\nu} V(\mathbf{x})$

for all $\beta>0$ and some $\nu \neq 0$ or -2 . In place of (7) consider

$\mathbf{x}(t)=\alpha^{2} \mathbf{x}_{0}\left(\alpha^{\nu-2} t\right), \quad \mathbf{p}(t)=\alpha^{\nu} \mathbf{p}_{0}\left(\alpha^{\nu-2} t\right)$.

This is a solution of (3) and (4) with

$$
\begin{gathered}
\lambda=\lambda_{0}, \quad E=\alpha^{2 \nu} E_{0}, \\
T=\alpha^{2-\nu} T_{0}, \quad S=\alpha^{\nu+2} S_{0}, \\
\rho_{\gamma}(E)=a_{\gamma} \sin \left[\frac{S_{0}}{\hbar}\left(\frac{E}{E_{0}}\right)^{\frac{\nu+2}{2 \nu}}+\eta_{\gamma}\right] .
\end{gathered}
$$

In this construction (known for a long time) the orbits for different values of $\alpha$ are not the same, but they are geometrically similar. The variable with respect to which the spectral oscillations take place is a peculiar power of $E$, and the analogue of the characteristic length $l_{\gamma}$ is a similarly complicated (but $\alpha$-invariant) function of $S_{0}$ and $E_{0}$.

Proposition 3 (Cf. [13].) Suppose that

$V_{1}(\beta \mathbf{x})=\beta^{\nu_{1}} V_{1}(\mathbf{x}), \quad V_{2}(\beta \mathbf{x})=\beta^{\nu_{2}} V_{2}(\mathbf{x})$.

Perform the scaling (11) appropriate to $V_{1}$. Then $(\mathbf{x}(t), \mathbf{p}(t))$ solves (3) and (4) with

$$
\begin{gathered}
\lambda_{1}=\lambda_{10}, \quad \lambda_{2}=\alpha^{2\left(\nu_{1}-\nu_{2}\right)} \lambda_{20}, \\
E=\alpha^{2 \nu_{1}} E_{0},
\end{gathered}
$$

and (12) and (13) hold with $\nu=\nu_{1}$.

The point of this transformation is to associate a fixed orbit with a one-parameter family of classical situations in which one coupling constant is held fixed but the other varies with energy. The prototype [8] is the Hamiltonian of a hydrogen atom in a constant magnetic field,

$H(\mathbf{x}, \mathbf{p})=\frac{\mathbf{p}^{2}}{2 m}-\frac{e^{2}}{r}+\frac{e^{2}}{8 m c^{2}} B^{2}\left(x^{2}+y^{2}\right)$.

The magnetic field is a continuous variable that is under the experimenter's control; the charge of the proton is not! Therefore, to get a family of experimentally realizable systems one applies Proposition 3 with $\nu_{1}=-1, \nu_{2}=2$.

In principle Proposition 3 can be extended to an arbitrary potential in the role of $V_{2}$. Instead of a scaling of $\lambda_{2}$ as in (15), one obtains a oneparameter family of potentials

$V_{2[\alpha]}(\mathbf{x}) \equiv \alpha^{2 \nu_{1}} V_{2}\left(\alpha^{-2} \mathbf{x}\right)$,

not related among themselves by a simple homogeneity. (Of course, experimental realization of such a family would be more difficult than just adjusting the strength of an applied magnetic or electric field.)

A great advantage of identifying closed orbits that remain fixed over the entire range of $E$ is a clarification of the discussion of "semiclassical limit". Each orbit identifies a family of situations that are equivalent classically but distinct quantum-mechanically. A semiclassical regime is 
reached by moving along a family in the direction of increasing $S$. In units with $\hbar=1=2 m$, the dimension of $\omega \equiv \sqrt{E}$ is inverse length, and the quantum parameter (tantamount to $S / \hbar$ ) is the product of $\omega$ with some length characteristic of the system. The latter makes sense also in classical wave theories (the geometrical optics limit), as in $[1,9]$.

The regime of large action is usually also that of high energy, but when homogeneity scaling (Proposition 2 or 3 ) has been applied with $0>$ $\nu>-2$, the semiclassical limit is that of decreasing $|E|$, because of the negative exponent in (13). This includes in particular the Coulomb potential, where (for $\lambda$ fixed) the regime of "large quantum numbers" is reached as $E$ approaches 0 from below.

To conclude, let us place these scaling considerations into the context of the overall enterprise of relating spectral properties to geometrical ones, using as intermediaries the various integral kernels associated with $H$. Here $H$ is a differential operator (say second-order, self-adjoint, positive), and "geometrical" is meant in the broadest sense, to include the potential functions in $H$ and even the spectrum of orbits of the corresponding classical system. Classic inverse theory, particularly that based on the heat kernel, deduces geometrical information from spectral data at the semiclassical end of the spectrum; the main point of the heat kernel expansion is that the fine structure of the eigenvalue distribution washes out and the gross high-energy asymptotics by itself retrodicts the basic geometry. But when periodic-orbit theory is used to predict spectra, only the lowest eigenvalues are easily obtained; as energy increases, an exponentially growing number of increasingly long orbits must be included to get good resolution of the spectrum. In the physics literature this has often been considered surprising or paradoxical, since semiclassical methods were expected to be most accurate at large energies (or actions).

I propose that this phenomenon is merely an instance of the familiar principle that when a calculation is very stable and involves some kind of smoothing or averaging, the inverse calculation is likely to be unstable (highly dependent on the details of the input) and hence difficult. (Solving a Fredholm integral equation of the first kind — or inverting any compact operator - is a precise mathematical example.) In the semiclassical regime the stable direction of prediction is from the spectrum to the geometry; in the opposite regime the stable direction is from the geometry to the spectrum. Rather than call that the "deep quantum regime", as if the duality between the quantum eigenvalues and the classical orbits were irrelevant there, we might better say "resonant regime". There, resonant behavior emerges on the spectral side, out of the geometry and classical mechanics, much as, in the opposite "classical regime", classical-mechanical behavior emerges on the geometrical side, out of the quantum substrate.

\section{REFERENCES}

1. R. Balian and C. Bloch, Ann. Phys. (NY) 60 (1970) 401.

2. F. H. Brownell, Pacific J. Math. 5 (1955) 483.

3. L. Hörmander, Acta Math. 121 (1968) 193.

4. R. Estrada and S. A. Fulling, Electron. J. Diff. Eqs. 1999 (1999), No. 7.

5. S. A. Fulling, to be published, quant-ph/ 0012070.

6. J. B. Delos and M.-L. Du, IEEE J. Quantum Electron. 245 (1988) 1445.

7. M. Combescure, J. Ralston, and D. Robert, Commun. Math. Phys. 202 (1999) 463.

8. H. Friedrich and D. Wintgen, Phys. Reports 183 (1989) 37.

9. R. Balian and C. Bloch, Ann. Phys. (NY) 69 (1972) 76 .

10. J. J. Duistermaat and V. W. Guillemin, Invent. Math. 29 (1975) 39.

11. M. C. Gutzwiller, J. Math. Phys. 12 (1971) 343.

12. J. Main, C. Jung, and H. S. Taylor, J. Chem. Phys. 107 (1997) 6577.

13. H. Friedrich, in Atoms and Molecules in Strong External Fields, ed. by P. Schmelcher and W. Schweizer, Plenum, New York, 1998, pp. $153-167$. 